\title{
Association Between Polycyclic Aromatic Hydrocarbons and Urinary Incontinence in U.S. Adult: NHANES 2007-2016
}

\section{Zilong Zhang}

Sichuan University West China Hospital Department of Urology

Kun Jin

Sichuan University West China Hospital Department of Urology

Shi Qiu

Sichuan University West China Hospital Department of Urology

Xinyi Huang

Sichuan University No 4 West China Teaching Hospital: Sichuan University West China School of Public Health

Dan Hu

Sichuan University West China Hospital

\section{Zhongyuan Jiang}

Sichuan University West China Hospital

\section{Mingda Wang}

Sichuan University No 4 West China Teaching Hospital: Sichuan University West China School of Public Health

\section{Lu Yang}

Sichuan University West China Hospital Department of Urology

Tianhai Lin

Sichuan University West China Hospital Department of Urology

Xianghong Zhou

Sichuan University West China Hospital Department of Urology

Di Jin

Sichuan University West China Hospital Department of Urology

Yu Zhan

Sichuan University - Jiangan Campus

Qiang Wei ( $\nabla$ weiqiang163163@163.com )

Sichuan University West China Hospital Department of Urology https://orcid.org/0000-0002-3380-4932

\section{Research}

Keywords: Polycyclic aromatic hydrocarbons, urinary incontinence, NHANES

Posted Date: August 13th, 2021

DOI: https://doi.org/10.21203/rs.3.rs-783431/v1

License: (c) (i) This work is licensed under a Creative Commons Attribution 4.0 International License. Read Full License 


\section{Abstract}

Background: It have been reported that polycyclic aromatic hydrocarbons (PAHs) were associated with a variety of diseases, but it is still unclear that whether PAHs are associated with urinary incontinence (UI). This study aims to assess the association between PAHs and UI.

Methods: Data were collected from the National Health and Nutrition Examination Survey (NHANES) from 2007 to 2016.10 PAHs were detected totally. UI was diagnosed and distinguished into stress UI (SUI), urgency UI (UUI), and mixed UI (MUI) by answers of self-reported questions. Multivariate logistic regression analyses categorizing PAHs as quartiles were performed to detect the correlation between urinary PAHs and all three Ul subtypes. Models were adjusted for common epidemiological factors including demographic and living habits factors.

Results: A total of 12,393 participants of NHANES with available urine PAHs and UI data were eligible. No significant differences were observed between characteristics of participants with and without UI. Increasing prevalence of UUI was associated with the highest quantiles of 1-hydroxynapthalene ( $\mathrm{OR}=1.208,95 \% \mathrm{Cl}=1.026-1.422, \mathrm{p}$ for trend=0.036), 1-hydroxyphenanthrene $(\mathrm{OR}=1.203,95 \% \mathrm{Cl}=1.017-1.423, \mathrm{p}$ for trend=0.004), and 1-hydroxypyrene $(\mathrm{OR}=1.251,95 \% \mathrm{Cl}=1.062-1.475, \mathrm{p}$ for trend=0.013) compared with the lowest quantiles. Increasing rate of SUI was observed in participants with higher exposure of all 9 PAHs ( $p$ for trend<0.025) except 3-hydroxyfluorene $(\mathrm{OR}=1.115,95 \% \mathrm{Cl}=0.929-1.338, \mathrm{p}$ for trend=0.241). Compared with the lowest quantiles, association between $\mathrm{MUI}$ and the highest quantiles of 1-hydroxyphenanthrene $(\mathrm{OR}=1.554,95 \% \mathrm{Cl}=1.244-1.942$, $\mathrm{p}$ for trend<0.001) and 1-hydroxypyrene $(\mathrm{OR}=1.307,95 \% \mathrm{Cl}=1.047-1.630, \mathrm{p}$ for trend=0.018) was observed.

Conclusion: Our study suggested a positive relationship between U-PAHs and the prevalence of all three types of UI. Almost all PAHs are positively correlated with SUI.

\section{Introduction}

Polycyclic Aromatic Hydrocarbons (PAHs) are organic substances distributed in air, soil, water and even food. They are composed of aromatic rings and consist of carbon as well as hydrogen atoms $(1 ; 2)$. PAHs are mainly generated by the incomplete combustion of organic matters (3). Automobile exhaust, fossil fuels using, tobacco smoking, asphalt production, and agricultural burning are the main ways of human exposure to PAHs (4). There have been studies assessing relationships between PAHs exposure and health problems, and some of them showed that PAHs may have carcinogenesis, teratogenesis, genotoxicity, immune abnormality and other adverse effects on human body (5-8).

Urine incontinence $(\mathrm{UI})$ is described as an involuntary loss of urine, which often increases with age (9). According to the publications, the involuntary urine loss can occur in up to $69 \%$ women as well as $39 \%$ men, which stands as an important factor in the overall health and quality of life (10-13). As the population ages, the medical costs associated with Ul are placing a huge burden on society (14). Thus, there has been a flurry of research in recent years focusing on the factors associated with UI. UI is commonly categorized into stress UI (SUI), urgency UI (UUI), and mixed UI (MUI).

UI has been revealed with a strong relation with sex hormones by a number of previous studies. $(15 ; 16)$ Meanwhile, a recent evidence suggested that PAHs were also associated with sex hormones. (17) To the best of my knowledge, few results on the relationship between PAH exposure and UI have been reported. The present study assessed the relationship between urinary PAHs and UI in the United States general population.

\section{Methods}

Study Design and Population

The present study used data from five cycles (2007-2016) of National Health and Nutrition Examination Survey (NHANES) (http://www.cdc.gov/nchs/nhanes.htm). NHANES collects demographic, behavioral, clinical, social, dietary, and laboratory data from non-institutional people in the United States, which is a cross-sectional survey of a representative population of the nation 
with purpose of making assessment of the health and nutrition condition of adults and minors across the country. The survey is programmed of National Center for Health Statistics (NCHS). Detailed information on participants' demographics, dietary factors, socioeconomic status, and medical status was collected in-home by professional interviewers. Standardized medical physical examinations as well as the biospecimen collection were performed by experienced medical personnel in mobile examination centers (MECs). Patients without laboratory data of urinary PAHs metabolites and without Ul questionnaire information were excluded. The study was approved by Ethics Review Board of NCHS, and all participants had been required to sign an informed consent form before the study began.

\section{Polycyclic Aromatic Hydrocarbons}

Through the survey, urine samples were collected by professionally trained technicians and then frozen in $-20^{\circ} \mathrm{C}$ environment according to Laboratory/Medical Procedures pending further analysis. PAHs in urine samples were tested using capillary gas chromatography combined with high resolution mass spectrometry (GC-HRMS), isotope dilution gas chromatography/tandem mass spectrometry (GC-MS/MS), and isotope dilution online solid phase extraction high performance liquid chromatography/tandem mass spectrometry (online SPE-HPLC-MS/MS). The specific test method varied with the year. (http://www.cdc.gov/nchs/nhanes.htm). Ten hydroxylated urinary PAH, namely, 1-hydroxynapthalene, 2-hydroxynapthalene, 2chydroxyfluorene, 3-hydroxyfluorene, 9-hydroxyfluorene, 1-hydroxypyrene, 1-hydroxyphenanthrene, 2-hydroxyphenanthrene, 3hydroxyphenanthrene, and 4-hydroxyphenanthrene were observed.

\section{Outcome Information}

Ul was determined and classified by following questionnaires among all participants over the age of 20. Participants with positive answer to "Have you leaked or lost control of even a small amount of urine with activity like coughing, lifting, or exercise during the past 12 months?" were categorized as SUI. UUI was diagnosed as answering yes to "Have you leaked or lost control of even a small amount of urine with an urge or pressure to urinate and you couldn't get to the toilet fast enough during the past 12 months?" People with answer YES to both UUI and SUI questions were diagnosed with MUI. The above questions were asked at MECs by trained interviews, using the Computer-Assisted Personal Interview system.

\section{Covariates Measurement}

The covariates adjusting association between urinary PAHs and UI include age, race (Mexican Hispanic, other Hispanic, nonHispanic white, non-Hispanic black, and others), body mass index (BMI), ratio of family income-poverty, marital status (living alone and married or living with partner and married or living with partner), educational level (under high school, high school or general educational development, and above high school), physical activity level (under moderate, moderate, and vigorous), Charlson comorbidity index, smoking status (never, former, and current), and alcohol drinking level (none, moderate, heavy). Smoking level was classified as never smoking ( $<100$ cigarettes during the life), former smoking ( $\geq 100$ cigarettes during the life, currently not smoking) and current smoking ( $\geq 100$ cigarettes during the life, currently smoking). The alcohol consumption categories were defined as none ( $0 \mathrm{~g}$ per day), moderate (0.1-27.9 g per day for men and 0.1-13.9 $\mathrm{g}$ per day for women), and heavy (no less than $28.0 \mathrm{~g}$ per day for men and no less than $14.0 \mathrm{~g}$ per day for women). All information is available on NHANES website (http://www.cdc.gov/nchs/nhanes.htm).

\section{Statistical Analysis}

The characteristics of participants were presented in the form of numbers (percentage) for categorical variables and mean \pm standard deviation (SD) for continuous variables. Differences between base line characteristics of people with UI and without UI were calculated using either Kruscal Whallis $\mathrm{H}$ test on continuous variables or a weighted chi-square test on categorical variables.

Since the concentrations of PAHs were diluted by urine, we adjusted it and used the creatinine corrected PAHs concentrations instead of the original concentrations for analysis. Because the urine PAHs showed a highly skew distribution, log transformation was performed then. Due to the same reason, PAHs levels were presented as geometric mean (GM), median, 
upper and lower quartiles. When the urine PAHs concentrations is lower than the lower limit of detection (LLOD), it is replaced by the square root of 2. The continuous variables of urine PAHs concentrations were separated into four ranges by the median, upper and lower quartiles, and were transformed into categorical variables. Multivariable logistic regression models were used and the relationships between urinary PAHs and three $\mathrm{UI}$ subtypes were estimated with odds ratios (ORs) and corresponding $95 \%$ confidence intervals $(95 \% \mathrm{Cl})$. Three models were established: a non-adjusted model (Model I), a partially adjusted model (Model II) with age, race, and BMI adjusted, and a fully adjusted model (Model III) with age, race, BMI, ratio of family incomepoverty, educational level, marital status, physical activity, Chalson comorbidity index, smoking status, and alcohol drinking adjusted.

Missing values were entered by the median of existing continuous variables or by mode of existing categorical variables. All statistical analyses were performed with R (http:// www.R-project.org, The R Foundation) and Empower (www.empowerstats.com). Considering the complex sampling design of NHANES, weighted analyses were conducted. The pvalues of multiple testing were all adjusted and statistically significant were defined as two-tailed $p<0.05$.

\section{Results}

In the NHANES database from 2007 to 2016, a total of 50,588 participants aged over 20 were enrolled in the survey, among which 13,085 participants with detectable level of urinary PHAs metabolites were included. 692 participants were excluded due to lack of UI information. Finally, a total of 12,393 adults over 20 years of age were analyzed. Figure 1 showed the flowchart of participants selection.

Table 1 presented the basic characteristics of all participants in groups with $\mathrm{UI}$ and without Ul. Significant differences in age, gender, race, ration of family income-poverty, educational level, marital, BMI, Charlson comorbidity index, and alcohol drinking were observed between participants with and without UI. Table 2 presented the distribution of detectable urinary PAHs, including GM, median and quartiles (Q1, Q2) for each . 
Table 1

Basic characteristics of participants in the US adults, NHANES $2007 \sim 2016$

\begin{tabular}{|c|c|c|c|}
\hline Characteristics $(n=12,393)$ & UI $(n=4,229)$ & Without UI $(n=8,164)$ & P value \\
\hline Age, mean \pm SD (years) & $55.62 \pm 16.88$ & $45.99 \pm 17.86$ & $<0.001$ \\
\hline Gender, n (\%) & & & $<0.001$ \\
\hline Male & $1051(24.85 \%)$ & $5104(62.52 \%)$ & \\
\hline Female & $3178(75.15 \%)$ & $3060(37.48 \%)$ & \\
\hline Race, n (\%) & & & $<0.001$ \\
\hline Mexican American & $739(17.47 \%)$ & $1369(16.77 \%)$ & \\
\hline Other Hispanic & $313(7.40 \%)$ & $692(8.48 \%)$ & \\
\hline Non-Hispanic White & $2061(48.73 \%)$ & $3678(45.05 \%)$ & \\
\hline Non-Hispanic Black & $861(20.36 \%)$ & $1661(20.35 \%)$ & \\
\hline Other Race & $255(6.03 \%)$ & $764(9.36 \%)$ & \\
\hline Ratio of Family Income to Poverty, n (\%) & & & 0.024 \\
\hline$<1.3$ & $1227(29.01 \%)$ & $2286(28.00 \%)$ & \\
\hline $1.3 \sim 3.5$ & $1804(42.66 \%)$ & $3372(41.30 \%)$ & \\
\hline$>3.5$ & $1198(28.33 \%)$ & $2506(30.70 \%)$ & \\
\hline Education Level, n (\%) & & & $<0.001$ \\
\hline Less than High School & $1198(28.33 \%)$ & $2036(24.94 \%)$ & \\
\hline High School/GED & $994(23.50 \%)$ & $1864(22.83 \%)$ & \\
\hline Above High School & $2037(48.17 \%)$ & $4264(52.23 \%)$ & \\
\hline Marital, n (\%) & & & 0.001 \\
\hline Married or Living with Partner & $2479(58.62 \%)$ & $5030(61.61 \%)$ & \\
\hline Living Alone & $1750(41.38 \%)$ & $3134(38.39 \%)$ & \\
\hline BMI, n (\%) & & & $<0.001$ \\
\hline$\leqq 25$ & $1031(24.38 \%)$ & $2659(32.57 \%)$ & \\
\hline $25 \sim 30$ & $1297(30.67 \%)$ & $2843(34.82 \%)$ & \\
\hline$>30$ & $1901(44.95 \%)$ & $2662(32.61 \%)$ & \\
\hline Charlson Comorbidity Index, n (\%) & & & $<0.001$ \\
\hline 0 & $1951(46.13 \%)$ & $5323(65.20 \%)$ & \\
\hline 1 & $1560(36.89 \%)$ & $2199(26.94 \%)$ & \\
\hline $2,3,4$, and 5 & $718(16.98 \%)$ & $642(7.86 \%)$ & \\
\hline Smoking, n (\%) & & & 0.241 \\
\hline
\end{tabular}

abbr. UI, urinary incontinence; GED, general educational development; BMI, body mass index; SD, standard deviation; $n$, number of subjects; \%, weighted percentage

a Difference in characteristics between participants with and without UI 


\begin{tabular}{|c|c|c|c|}
\hline Characteristics $(n=12,393)$ & Ul $(n=4,229)$ & Without UI $(n=8,164)$ & P value \\
\hline Never & $2233(52.80 \%)$ & $4398(53.87 \%)$ & \\
\hline Former & $1272(30.08 \%)$ & $2337(28.63 \%)$ & \\
\hline Current & $724(17.12 \%)$ & $1429(17.50 \%)$ & \\
\hline Alcohol Drinking, n (\%) & & & $<0.001$ \\
\hline None & $3441(81.37 \%)$ & $6024(73.79 \%)$ & \\
\hline Moderate & $262(6.20 \%)$ & $793(9.71 \%)$ & \\
\hline Heavy & $526(12.44 \%)$ & $1347(16.50 \%)$ & \\
\hline \multicolumn{4}{|c|}{$\begin{array}{l}\text { abbr. UI, urinary incontinence; GED, general educational development; BMI, body mass index; SD, standard deviation; } n \text {, } \\
\text { number of subjects; \%, weighted percentage }\end{array}$} \\
\hline
\end{tabular}

Table 2

Distribution of urinary PAH levels (ng/L) in US adults, NHANES $2007 \sim 2016$

\begin{tabular}{|lllll|}
\hline U-PAHs & GM & Q1 & Median & Q3 \\
\hline 1-Hydroxynapthalene & 31032.023 & 787.00 & 1890.00 & 6470.00 \\
\hline 2-Hydroxynapthalene & 8434.22 & 1730.78 & 4090.00 & 9846.27 \\
\hline 3-Hydroxyfluorene & 320.18 & 41.00 & 85.50 & 260.00 \\
\hline 2-Hydroxyfluorene & 628.55 & 114.73 & 237.00 & 596.00 \\
\hline 3-Hydroxyphenanthrene & 211.30 & 43.00 & 86.60 & 178.72 \\
\hline 1-Hydroxyphenanthrene & 214.97 & 68.40 & 130.00 & 242.00 \\
\hline 2-Hydroxyphenanthrene & 113.08 & 33.73 & 65.20 & 126.00 \\
\hline 1-Hydroxypyrene & 198.17 & 49.50 & 94.00 & 199.00 \\
\hline 9-Hydroxyfluorene & 602.18 & 144.00 & 299.65 & 625.47 \\
\hline 4-Hydroxyphenanthrene & 44.23 & 12.00 & 25.00 & 50.08 \\
\hline abbr. GM, geometric mean; Q1, quartile 1; Q3, quartile 3. & \\
\hline
\end{tabular}

Table 3 showed the association between 10 PAHs and UUI and the results of trend test. Significantly increasing UUI prevalence was associated with the highest quantiles compared with the lowest quantiles of 1-hydroxynapthalene $(\mathrm{OR}=1.208,95 \% \mathrm{Cl}=$ 1.026-1.422, $\mathrm{p}$ for trend $=0.036), 1$-hydroxyphenanthrene $(\mathrm{OR}=1.203,95 \% \mathrm{Cl}=1.017-1.423, \mathrm{p}$ for trend $=0.004)$, and 1 hydroxypyrene $(\mathrm{OR}=1.215,95 \% \mathrm{Cl}=1.062-1.475$, $\mathrm{p}$ for trend $=0.013)$ in Model III. The association between PAHs and SUI and trend test were presented in Table 4. In Model III, significantly increases of SUl odds were observed in all PAHs ( $p$ for trend all lower than 0.025 ) except 3-hdroxyfluorene $(O R=1.115,95 \% \mathrm{Cl}=0.020-1.338, \mathrm{p}$ for trend $=0.241)$. Table 5 showed the results of analysis of association between PAHs categorized as quartiles and MUl. Compared with the lowest quantile, positive association between the highest quantile of 1-hydroxyphenanthrene $(\mathrm{OR}=1.554,95 \% \mathrm{Cl}=1.244-1.942, \mathrm{p}$ for trend $<0.001)$ and 1-hydroxypyrene $(\mathrm{OR}=1.307,95 \% \mathrm{Cl}=1.047-1.630, \mathrm{p}$ for trend $=0.018)$ and $\mathrm{MUI}$ was observed in the fully adjusted model. 
Table 3

Association between urinary PAHs and UUI among US adults, NHANES 2007 2016

\begin{tabular}{|c|c|c|c|c|c|c|}
\hline \multirow[t]{2}{*}{ Exposure } & \multicolumn{2}{|l|}{ Model la } & \multicolumn{2}{|l|}{ Model II } & \multicolumn{2}{|l|}{ Model IIIC } \\
\hline & OR $(95 \% \mathrm{Cl})$ & $\begin{array}{l}p \\
\text { value }\end{array}$ & OR $(95 \% \mathrm{Cl})$ & $\begin{array}{l}p \\
\text { value }\end{array}$ & OR (95\%Cl) & $\begin{array}{l}p \\
\text { value }\end{array}$ \\
\hline \multicolumn{7}{|c|}{ 1-hydroxynapthalene } \\
\hline quantile 1 & 1.000 & - & 1.000 & - & 1.000 & - \\
\hline quantile 2 & $1.025(0.904,1.162)$ & 0.701 & $1.076(0.938,1.235)$ & 0.297 & $1.061(0.924,1.219)$ & 0.403 \\
\hline quantile 3 & $1.110(0.981,1.256)$ & 0.098 & $1.141(0.995,1.309)$ & 0.059 & $1.100(0.955,1.266)$ & 0.187 \\
\hline quantile 4 & $1.174(1.038,1.328)$ & 0.011 & $1.394(1.209,1.607)$ & $\begin{array}{l}< \\
0.001\end{array}$ & $1.208(1.026,1.422)$ & 0.023 \\
\hline $\mathrm{p}$ for trend & & 0.010 & & $\begin{array}{l}< \\
0.001\end{array}$ & & 0.036 \\
\hline
\end{tabular}

2-hydroxynapthalene

\begin{tabular}{|c|c|c|c|c|c|c|}
\hline quantile 1 & 1.000 & - & 1.000 & - & 1.000 & - \\
\hline quantile 2 & $0.877(0.777,0.991)$ & 0.035 & $1.021(0.893,1.168)$ & 0.758 & $0.967(0.843,1.108)$ & 0.628 \\
\hline quantile 3 & $0.909(0.805,1.025)$ & 0.120 & $1.237(1.075,1.422)$ & 0.003 & $1.108(0.958,1.281)$ & 0.168 \\
\hline quantile 4 & $0.853(0.755,0.964)$ & 0.011 & $1.325(1.140,1.540)$ & $\begin{array}{l}< \\
0.001\end{array}$ & $1.063(0.893,1.264)$ & 0.492 \\
\hline$p$ for trend & & 0.054 & & $\begin{array}{l}< \\
0.001\end{array}$ & & 0.416 \\
\hline
\end{tabular}

3-hydroxyfluorene

\begin{tabular}{lllllll} 
quantile 1 & 1.000 & - & 1.000 & - & 1.000 & - \\
\hline quantile 2 & $0.852(0.756,0.960)$ & 0.008 & $0.981(0.856,1.123)$ & 0.776 & $0.953(0.831,1.092)$ & 0.487 \\
quantile 3 & $0.733(0.649,0.829)$ & $<$ & $0.974(0.843,1.126)$ & 0.725 & $0.911(0.785,1.057)$ & 0.217 \\
& & 0.001 & & & & \\
quantile 4 & $0.788(0.698,0.889)$ & $<$ & $1.204(1.038,1.396)$ & 0.014 & $0.940(0.777,1.137)$ & 0.521 \\
& & 0.001 & & & & 0.896 \\
p for trend & & 0.020 & & 0.001 & &
\end{tabular}

2-hydroxyfluorene

\begin{tabular}{|lllllll|} 
quantile 1 & 1.000 & - & 1.000 & - & 1.000 & - \\
\hline quantile 2 & $0.919(0.815,1.035)$ & 0.164 & $1.086(0.947,1.246)$ & 0.236 & $1.032(0.898,1.186)$ & 0.654
\end{tabular}

abbr. PAH, polycyclic aromatic hydrocarbons; UUl, urgency urinary incontinence; OR, odds ratio; $\mathrm{Cl}$, confidence interval

a. Model I: unadjusted model

b. Model II: adjusted for age $(<60, \geqq 60)$, race (Mexican American, other Hispanic, non-Hispanic white, non-Hispanic black, other races), and $\mathrm{BMI}(\leqq 25,25 \sim 30,>30)$.

c. Model III: adjusted for age $(<60, \geqq 60)$, race (Mexican American, other Hispanic, non-Hispanic white, non-Hispanic black, other races), $\mathrm{BMI}(\leqq 25,25 \sim 30,>30)$, ratio of family income to poverty $(<1.3,1.3 \sim 3.5,>3.5)$, education level (less than high school, high school or general educational development), marital status (married or living with partner, living alone), Charlson comorbidity index $(0,1,2345)$, physical activity (less than moderate, moderate, vigorous), smoking status (never, former, current), alcohol drinking (none, moderate, heavy). 


\begin{tabular}{|c|c|c|c|c|c|c|}
\hline \multirow[t]{2}{*}{ Exposure } & \multicolumn{2}{|l|}{ Model $\left.\right|^{\mathrm{a}}$} & \multicolumn{2}{|l|}{ Model IIb } & \multicolumn{2}{|l|}{ Model IIIC } \\
\hline & OR (95\%Cl) & $\begin{array}{l}p \\
\text { value }\end{array}$ & OR $(95 \% \mathrm{Cl})$ & $\begin{array}{l}p \\
\text { value }\end{array}$ & OR (95\%Cl) & $\begin{array}{l}p \\
\text { value }\end{array}$ \\
\hline quantile 3 & $0.779(0.690,0.881)$ & $\begin{array}{l}< \\
0.001\end{array}$ & $1.045(0.902,1.210)$ & 0.562 & $0.953(0.818,1.110)$ & 0.535 \\
\hline quantile 4 & $0.835(0.740,0.943)$ & 0.004 & $1.294(1.111,1.507)$ & $\begin{array}{l}< \\
0.001\end{array}$ & $1.005(0.832,1.215)$ & 0.957 \\
\hline$p$ for trend & & 0.030 & & $\begin{array}{l}< \\
0.001\end{array}$ & & 0.937 \\
\hline
\end{tabular}

3-

hydroxyphenanthrene

$\begin{array}{rllllll}\text { quantile 1 } & 1.000 & - & 1.000 & - & 1.000 & - \\ \text { quantile 2 } & 0.861(0.749,0.989) & 0.035 & 1.017(0.870,1.189) & 0.832 & 0.963(0.822,1.129) & 0.645 \\ \text { quantile 3 } & 0.696(0.602,0.804) & < & 0.931(0.784,1.104) & 0.409 & 0.839(0.703,1.000) & 0.051 \\ & & 0.001 & & & & \\ \text { quantile 4 } & 0.823(0.715,0.948) & 0.007 & 1.238(1.034,1.483) & 0.020 & 1.016(0.833,1.240) & 0.875 \\ \text { p for trend } & & 0.044 & & 0.003 & & 0.408\end{array}$

$1-$

hydroxyphenanthrene

\begin{tabular}{|c|c|c|c|c|c|c|}
\hline quantile 1 & 1.000 & - & 1.000 & - & 1.000 & - \\
\hline quantile 2 & $0.865(0.766,0.976)$ & 0.019 & $0.999(0.870,1.146)$ & 0.984 & $0.964(0.839,1.108)$ & 0.605 \\
\hline quantile 3 & $0.857(0.759,0.968)$ & 0.013 & $1.149(0.989,1.335)$ & 0.069 & $1.083(0.930,1.262)$ & 0.304 \\
\hline quantile 4 & $0.880(0.780,0.993)$ & 0.038 & $1.333(1.134,1.566)$ & $\begin{array}{l}< \\
0.001\end{array}$ & $1.203(1.017,1.423)$ & 0.031 \\
\hline$p$ for trend & & 0.162 & & $\begin{array}{l}< \\
0.001\end{array}$ & & 0.004 \\
\hline
\end{tabular}

2-

hydroxyphenanthrene

\begin{tabular}{|lllllll|} 
quantile 1 & 1.000 & - & 1.000 & - & 1.000 & - \\
\hline quantile 2 & $0.907(0.788,1.045)$ & 0.176 & $1.137(0.970,1.333)$ & 0.113 & $1.079(0.918,1.269)$ & 0.354 \\
\hline quantile 3 & $0.867(0.752,0.999)$ & 0.049 & $1.241(1.043,1.476)$ & 0.015 & $1.131(0.946,1.352)$ & 0.177 \\
\hline quantile 4 & $0.815(0.707,0.941)$ & 0.005 & $1.291(1.071,1.556)$ & 0.007 & $1.090(0.892,1.332)$ & 0.400 \\
\hline p for trend & & 0.009 & & 0.025 & & 0.704
\end{tabular}

abbr. PAH, polycyclic aromatic hydrocarbons; UUl, urgency urinary incontinence; OR, odds ratio; $\mathrm{Cl}$, confidence interval

a. Model I: unadjusted model

b. Model II: adjusted for age $(<60, \geqq 60)$, race (Mexican American, other Hispanic, non-Hispanic white, non-Hispanic black, other races), and $\mathrm{BMI}(\leqq 25,25 \sim 30,>30)$.

c. Model III: adjusted for age $(<60, \geqq 60)$, race (Mexican American, other Hispanic, non-Hispanic white, non-Hispanic black, other races), BMI ( $\leqq 25,25 \sim 30,>30)$, ratio of family income to poverty $(<1.3,1.3 \sim 3.5,>3.5)$, education level (less than high school, high school or general educational development), marital status (married or living with partner, living alone), Charlson comorbidity index $(0,1,2345)$, physical activity (less than moderate, moderate, vigorous), smoking status (never, former, current), alcohol drinking (none, moderate, heavy). 


\begin{tabular}{|c|c|c|c|c|c|c|}
\hline \multirow[t]{2}{*}{ Exposure } & \multicolumn{2}{|l|}{ Model la } & \multicolumn{2}{|l|}{ Model II } & \multicolumn{2}{|l|}{ Model IIIC } \\
\hline & OR $(95 \% \mathrm{Cl})$ & $\begin{array}{l}\mathrm{p} \\
\text { value }\end{array}$ & OR $(95 \% \mathrm{Cl})$ & $\begin{array}{l}\text { p } \\
\text { value }\end{array}$ & OR $(95 \% \mathrm{Cl})$ & $\begin{array}{l}\text { p } \\
\text { value }\end{array}$ \\
\hline \multicolumn{7}{|l|}{ 1-hydroxypyrene } \\
\hline quantile 1 & 1.000 & - & 1.000 & - & 1.000 & - \\
\hline quantile 2 & $0.884(0.784,0.996)$ & 0.043 & $1.091(0.959,1.241)$ & 0.185 & $1.066(0.935,1.214)$ & 0.339 \\
\hline quantile 3 & $0.816(0.721,0.923)$ & 0.001 & $1.262(1.097,1.452)$ & 0.001 & $1.181(1.022,1.365)$ & 0.024 \\
\hline quantile 4 & $0.758(0.669,0.858)$ & $<.001$ & $1.446(1.243,1.681)$ & $<.001$ & $1.251(1.062,1.475)$ & 0.008 \\
\hline$p$ for trend & & $<.001$ & & $<.001$ & & 0.013 \\
\hline \multicolumn{7}{|c|}{ 9-hydroxyfluorene } \\
\hline quantile 1 & 1.000 & - & 1.000 & - & 1.000 & - \\
\hline quantile 2 & $0.884(0.759,1.030)$ & 0.114 & $0.988(0.834,1.171)$ & 0.892 & $0.938(0.789,1.114)$ & 0.463 \\
\hline quantile 3 & $0.847(0.726,0.988)$ & 0.035 & $1.047(0.873,1.255)$ & 0.624 & $0.941(0.780,1.135)$ & 0.524 \\
\hline quantile 4 & $0.869(0.746,1.013)$ & 0.073 & $1.149(0.948,1.394)$ & 0.158 & $0.915(0.737,1.136)$ & 0.423 \\
\hline$p$ for trend & & 0.192 & & 0.077 & & 0.575 \\
\hline \multicolumn{7}{|c|}{$\begin{array}{l}\text { 4- } \\
\text { hydroxyphenanthrene }\end{array}$} \\
\hline quantile 1 & 1.000 & - & 1.000 & - & 1.000 & - \\
\hline quantile 2 & $0.949(0.769,1.171)$ & 0.626 & $1.054(0.835,1.330)$ & 0.660 & $1.046(0.826,1.325)$ & 0.709 \\
\hline quantile 3 & $0.893(0.721,1.105)$ & 0.296 & $\begin{array}{l}1.095(0.853 \\
1.405)\end{array}$ & 0.477 & $1.072(0.829,1.387)$ & 0.596 \\
\hline quantile 4 & $0.843(0.679,1.045)$ & 0.119 & $1.181(0.901,1.548)$ & 0.228 & $1.090(0.812,1.463)$ & 0.566 \\
\hline$p$ for trend & & 0.121 & & 0.231 & & 0.645 \\
\hline \multicolumn{7}{|c|}{ abbr. PAH, polycyclic aromatic hydrocarbons; UUI, urgency urinary incontinence; OR, odds ratio; $\mathrm{Cl}$, confidence interval } \\
\hline \multicolumn{7}{|c|}{ a. Model I: unadjusted model } \\
\hline \multicolumn{7}{|c|}{$\begin{array}{l}\text { b. Model II: adjusted for age }(<60, \geqq 60) \text {, race (Mexican American, other Hispanic, non-Hispanic white, non-Hispanic black, } \\
\text { other races), and } \mathrm{BMI}(\leqq 25,25 \sim 30,>30) \text {. }\end{array}$} \\
\hline \multicolumn{7}{|c|}{$\begin{array}{l}\text { c. Model III: adjusted for age }(<60, \geqq 60) \text {, race (Mexican American, other Hispanic, non-Hispanic white, non-Hispanic black, } \\
\text { other races), BMI ( } \leqq 25,25 \sim 30,>30 \text {, ratio of family income to poverty }(<1.3,1.3 \sim 3.5,>3.5) \text {, education level (less than } \\
\text { high school, high school or general educational development), marital status (married or living with partner, living alone), } \\
\text { Charlson comorbidity index }(0,1,2345) \text {, physical activity (less than moderate, moderate, vigorous), smoking status (never, } \\
\text { former, current), alcohol drinking (none, moderate, heavy). }\end{array}$} \\
\hline
\end{tabular}


Table 4

Association between urinary PAHs and SUI among US adults, NHANES 2007 2016

\begin{tabular}{|c|c|c|c|c|c|c|}
\hline \multirow[t]{2}{*}{ Exposure } & \multicolumn{2}{|l|}{ Model la } & \multicolumn{2}{|l|}{ Model Ilb } & \multicolumn{2}{|l|}{ Model IIlc } \\
\hline & OR $(95 \% \mathrm{Cl})$ & $p_{\text {value }}$ & OR $(95 \% \mathrm{Cl})$ & $p_{\text {value }}$ & OR $(95 \% \mathrm{Cl})$ & $\begin{array}{l}p \\
\text { value }\end{array}$ \\
\hline \multicolumn{7}{|c|}{ 1-hydroxynapthalene } \\
\hline quantile 1 & 1.000 & - & 1.000 & - & 1.000 & - \\
\hline quantile 2 & $0.867(0.771,0.975)$ & 0.017 & $1.102(0.971,1.251)$ & 0.132 & $1.122(0.987,1.275)$ & 0.079 \\
\hline quantile 3 & $0.774(0.687,0.872)$ & $<.001$ & $0.993(0.873,1.130)$ & 0.914 & $1.043(0.912,1.193)$ & 0.538 \\
\hline quantile 4 & $\begin{array}{l}0.811(0.721 \\
0.913)\end{array}$ & $<.001$ & $1.204(1.053,1.377)$ & 0.007 & $1.266(1.082,1.481)$ & 0.003 \\
\hline$p$ for trend & & 0.025 & & 0.008 & & 0.006 \\
\hline \multicolumn{7}{|c|}{ 2-hydroxynapthalene } \\
\hline quantile 1 & 1.000 & - & 1.000 & - & 1.000 & - \\
\hline quantile 2 & $0.782(0.695,0.879)$ & $\dot{0} 001$ & $1.037(0.913,1.178)$ & 0.575 & $1.051(0.923,1.197)$ & 0.455 \\
\hline quantile 3 & $0.835(0.743,0.938)$ & 0.002 & $1.248(1.093,1.424)$ & 0.001 & $1.281(1.115,1.472)$ & $\dot{0} 0.001$ \\
\hline quantile 4 & $0.764(0.679,0.860)$ & $<.001$ & $1.376(1.194,1.587)$ & $\dot{0}_{0.001}$ & $1.454(1.233,1.714)$ & $\dot{0} .001$ \\
\hline $\mathrm{p}$ for trend & & 0.001 & & $<.001$ & & $\begin{array}{l}< \\
0.001\end{array}$ \\
\hline \multicolumn{7}{|l|}{ 3-hydroxyfluorene } \\
\hline quantile 1 & 1.000 & - & 1.000 & - & 1.000 & - \\
\hline quantile 2 & $0.733(0.654,0.823)$ & $<.001$ & $0.989(0.870,1.123)$ & 0.860 & $1.001(0.879,1.139)$ & 0.992 \\
\hline quantile 3 & $0.624(0.554,0.702)$ & $<.001$ & $0.993(0.867,1.138)$ & 0.921 & $1.020(0.886,1.174)$ & 0.781 \\
\hline quantile 4 & $0.622(0.553,0.700)$ & $<.001$ & $1.051(0.914,1.209)$ & 0.483 & $1.115(0.929,1.338)$ & 0.241 \\
\hline $\mathrm{p}$ for trend & & $\begin{array}{l}< \\
0.001\end{array}$ & & 0.314 & & 0.177 \\
\hline 2-hydroxyfluoren & & & & & & \\
\hline
\end{tabular}

abbr. PAH, polycyclic aromatic hydrocarbons; SUI, stress urinary incontinence; OR, odds ratio; $\mathrm{Cl}$, confidence interval

a. Model I: unadjusted model

b. Model II: adjusted for age $(<60, \geqq 60)$, race (Mexican American, other Hispanic, non-Hispanic white, non-Hispanic black, other races), and $\mathrm{BMI}(\leqq 25,25 \sim 30,>30)$.

c. Model III: adjusted for age $(<60, \geqq 60)$, race (Mexican American, other Hispanic, non-Hispanic white, non-Hispanic black, other races), $\mathrm{BMI}(\leqq 25,25 \sim 30,>30)$, ratio of family income to poverty $(<1.3,1.3 \sim 3.5,>3.5)$, education level (less than high school, high school or general educational development), marital status (married or living with partner, living alone), Charlson comorbidity index $(0,1,2345)$, physical activity (less than moderate, moderate, vigorous), smoking status (never, former, current), alcohol drinking (none, moderate, heavy). 


\begin{tabular}{|c|c|c|c|c|c|c|}
\hline \multirow[t]{2}{*}{ Exposure } & \multicolumn{2}{|l|}{ Model la } & \multicolumn{2}{|l|}{ Model Ilb } & \multicolumn{2}{|l|}{ Model IIIc } \\
\hline & OR $(95 \% \mathrm{Cl})$ & $\mathrm{p}_{\text {value }}$ & OR $(95 \% \mathrm{Cl})$ & $\begin{array}{l}\mathbf{p} \\
\text { value }\end{array}$ & OR $(95 \% \mathrm{Cl})$ & $\begin{array}{l}\mathrm{p} \\
\text { value }\end{array}$ \\
\hline quantile 1 & 1.000 & - & 1.000 & - & 1.000 & - \\
\hline quantile 2 & $0.765(0.682,0.859)$ & $\begin{array}{l}< \\
0.001\end{array}$ & $1.059(0.930,1.205)$ & 0.387 & $1.075(0.942,1.227)$ & 0.285 \\
\hline quantile 3 & $0.679(0.604,0.763)$ & $<0.001$ & $1.081(0.941,1.241)$ & 0.271 & $1.129(0.976,1.305)$ & 0.102 \\
\hline quantile 4 & $0.669(0.595,0.753)$ & $<0.001$ & $1.198(1.037,1.384)$ & 0.014 & $1.322(1.103,1.585)$ & 0.003 \\
\hline $\mathrm{p}$ for trend & & $<.001$ & & 0.015 & & 0.003 \\
\hline \multicolumn{7}{|c|}{$\begin{array}{l}\text { 3- } \\
\text { hydroxyphenanthrene }\end{array}$} \\
\hline quantile 1 & 1.000 & - & 1.000 & - & 1.000 & - \\
\hline quantile 2 & $0.855(0.748,0.977)$ & 0.021 & $1.193(1.029,1.383)$ & 0.019 & $1.201(1.034,1.397)$ & 0.017 \\
\hline quantile 3 & $0.722(0.630,0.829)$ & $<.001$ & $1.201(1.022,1.410)$ & 0.026 & $1.229(1.039,1.453)$ & 0.016 \\
\hline quantile 4 & $0.654(0.568,0.752)$ & $<0.001$ & $\begin{array}{l}1.278(1.074 \\
1.520)\end{array}$ & 0.006 & $1.346(1.111,1.631)$ & 0.002 \\
\hline $\mathrm{p}$ for trend & & $<0.001$ & & 0.044 & & 0.020 \\
\hline \multicolumn{7}{|c|}{$\begin{array}{l}\text { 1- } \\
\text { hydroxyphenanthrene }\end{array}$} \\
\hline quantile 1 & 1.000 & - & 1.000 & - & 1.000 & - \\
\hline quantile 2 & $0.772(0.686,0.869)$ & $<0.001$ & $1.123(0.984,1.282)$ & 0.084 & $1.144(1.001,1.308)$ & 0.048 \\
\hline quantile 3 & $0.804(0.716,0.904)$ & $<0.001$ & $1.437(1.245,1.659)$ & $<.001$ & $1.497(1.292,1.734)$ & $<.001$ \\
\hline quantile 4 & $0.805(0.716,0.904)$ & $<0.001$ & $1.718(1.470,2.009)$ & $<001$ & $1.816(1.543,2.137)$ & $<.001$ \\
\hline $\mathrm{p}$ for trend & & 0.017 & & $<.001$ & & $<.001$ \\
\hline \multicolumn{7}{|c|}{$\begin{array}{l}2- \\
\text { hydroxyphenanthrene }\end{array}$} \\
\hline quantile 1 & 1.000 & - & 1.000 & - & 1.000 & - \\
\hline \multicolumn{7}{|c|}{ abbr. PAH, polycyclic aromatic hydrocarbons; SUI, stress urinary incontinence; OR, odds ratio; $\mathrm{Cl}$, confidence interval } \\
\hline \multicolumn{7}{|c|}{ a. Model I: unadjusted model } \\
\hline \multicolumn{7}{|c|}{$\begin{array}{l}\text { b. Model II: adjusted for age }(<60, \geqq 60) \text {, race (Mexican American, other Hispanic, non-Hispanic white, non-Hispanic black, } \\
\text { other races), and } \mathrm{BMI}(\leqq 25,25 \sim 30,>30) \text {. }\end{array}$} \\
\hline \multicolumn{7}{|c|}{$\begin{array}{l}\text { c. Model III: adjusted for age }(<60, \geqq 60) \text {, race (Mexican American, other Hispanic, non-Hispanic white, non-Hispanic black, } \\
\text { other races), BMI ( } \leqq 25,25 \sim 30,>30) \text {, ratio of family income to poverty }(<1.3,1.3 \sim 3.5,>3.5) \text {, education level (less than } \\
\text { high school, high school or general educational development), marital status (married or living with partner, living alone), } \\
\text { Charlson comorbidity index }(0,1,2345) \text {, physical activity (less than moderate, moderate, vigorous), smoking status (never, } \\
\text { former, current), alcohol drinking (none, moderate, heavy). }\end{array}$} \\
\hline
\end{tabular}




\begin{tabular}{|c|c|c|c|c|c|c|}
\hline \multirow[t]{2}{*}{ Exposure } & \multicolumn{2}{|l|}{ Model la } & \multicolumn{2}{|l|}{ Model Ilb } & \multicolumn{2}{|l|}{ Model IIlc } \\
\hline & OR $(95 \% \mathrm{Cl})$ & $\begin{array}{l}p \\
\text { value }\end{array}$ & OR $(95 \% \mathrm{Cl})$ & $\begin{array}{l}p \\
\text { value }\end{array}$ & OR $(95 \% \mathrm{Cl})$ & $\begin{array}{l}p \\
\text { value }\end{array}$ \\
\hline quantile 2 & $0.768(0.671,0.879)$ & $\begin{array}{l}<.001 \\
0.0\end{array}$ & $1.049(0.903,1.218)$ & 0.532 & $1.056(0.907,1.230)$ & 0.484 \\
\hline quantile 3 & $0.700(0.611,0.803)$ & $\begin{array}{l}<.001 \\
0.0\end{array}$ & $1.124(0.954,1.325)$ & 0.161 & $1.141(0.963,1.353)$ & 0.127 \\
\hline quantile 4 & $0.694(0.605,0.796)$ & $\begin{array}{l}< \\
0.001\end{array}$ & $1.274(1.068,1.520)$ & 0.007 & $1.307(1.081,1.581)$ & 0.006 \\
\hline$p$ for trend & & $\begin{array}{l}< \\
0.001\end{array}$ & & 0.004 & & 0.003 \\
\hline
\end{tabular}

1-hydroxypyrene

\begin{tabular}{|c|c|c|c|c|c|c|}
\hline quantile 1 & 1.000 & - & 1.000 & - & 1.000 & - \\
\hline quantile 2 & $0.869(0.775,0.976)$ & 0.018 & $1.022(0.905,1.154)$ & 0.730 & $1.023(0.904,1.157)$ & 0.719 \\
\hline quantile 3 & $0.751(0.666,0.847)$ & $\begin{array}{l}< \\
0.001\end{array}$ & $1.125(0.985,1.286)$ & 0.083 & $1.147(0.999,1.317)$ & 0.051 \\
\hline quantile 4 & $0.687(0.608,0.776)$ & $\begin{array}{l}<.001 \\
0.00\end{array}$ & $1.247(1.080,1.439)$ & 0.003 & $1.286(1.099,1.505)$ & 0.002 \\
\hline$p$ for trend & & $\begin{array}{l}< \\
0.001\end{array}$ & & 0.001 & & $\begin{array}{l}< \\
0.001\end{array}$ \\
\hline
\end{tabular}

9-hydroxyfluorene

\begin{tabular}{|c|c|c|c|c|c|c|}
\hline quantile 1 & 1.000 & - & 1.000 & - & 1.000 & - \\
\hline quantile 2 & $0.801(0.692,0.928)$ & 0.003 & $1.051(0.895,1.234)$ & 0.546 & $1.074(0.911,1.266)$ & 0.394 \\
\hline quantile 3 & $0.735(0.633,0.853)$ & $\begin{array}{l}< \\
0.001\end{array}$ & $1.137(0.956,1.352)$ & 0.147 & $1.171(0.977,1.404)$ & 0.087 \\
\hline quantile 4 & $0.685(0.589,0.797)$ & $\begin{array}{l}< \\
0.001\end{array}$ & $1.208(1.003,1.454)$ & 0.047 & $1.268(1.030,1.562)$ & 0.025 \\
\hline$p$ for trend & & $\begin{array}{l}< \\
0.001\end{array}$ & & 0.049 & & 0.033 \\
\hline
\end{tabular}

4-

hydroxyphenanthrene

\begin{tabular}{|lllllll|} 
quantile 1 & 1.000 & - & 1.000 & - & 1.000 & - \\
quantile 2 & $0.932(0.758,1.145)$ & 0.501 & $1.273(1.015,1.597)$ & 0.037 & $1.320(1.048,1.662)$ & 0.019 \\
quantile 3 & $0.824(0.668,1.018)$ & 0.072 & $1.279(1.002,1.632)$ & 0.048 & $1.369(1.063,1.763)$ & 0.015 \\
\hline
\end{tabular}

abbr. PAH, polycyclic aromatic hydrocarbons; SUI, stress urinary incontinence; OR, odds ratio; $\mathrm{Cl}$, confidence interval

a. Model I: unadjusted model

b. Model II: adjusted for age $(<60, \geqq 60)$, race (Mexican American, other Hispanic, non-Hispanic white, non-Hispanic black, other races), and $\mathrm{BMI}(\leqq 25,25 \sim 30,>30)$.

c. Model III: adjusted for age $(<60, \geqq 60)$, race (Mexican American, other Hispanic, non-Hispanic white, non-Hispanic black, other races), BMI ( $\leqq 25,25 \sim 30,>30)$, ratio of family income to poverty $(<1.3,1.3 \sim 3.5,>3.5)$, education level (less than high school, high school or general educational development), marital status (married or living with partner, living alone), Charlson comorbidity index $(0,1,2345)$, physical activity (less than moderate, moderate, vigorous), smoking status (never, former, current), alcohol drinking (none, moderate, heavy). 


\begin{tabular}{|c|c|c|c|c|c|c|}
\hline \multirow[t]{2}{*}{ Exposure } & \multicolumn{2}{|l|}{ Model la } & \multicolumn{2}{|l|}{ Model Ilb } & \multicolumn{2}{|l|}{ Model IIlc } \\
\hline & OR $(95 \% \mathrm{Cl})$ & palue & OR $(95 \% \mathrm{Cl})$ & $\begin{array}{l}\text { p } \\
\text { value }\end{array}$ & OR $(95 \% \mathrm{Cl})$ & $\begin{array}{l}\mathrm{p} \\
\text { value }\end{array}$ \\
\hline quantile 4 & $0.883(0.716,1.088)$ & 0.242 & $1.664(1.281,2.162)$ & $<.001$ & $1.849(1.390,2.459)$ & $<.001$ \\
\hline$p$ for trend & & 0.322 & & $<.001$ & & $<.001$ \\
\hline \multicolumn{7}{|c|}{ abbr. PAH, polycyclic aromatic hydrocarbons; SUI, stress urinary incontinence; OR, odds ratio; $\mathrm{Cl}$, confidence interval } \\
\hline \multicolumn{7}{|c|}{ a. Model I: unadjusted model } \\
\hline \multicolumn{7}{|c|}{$\begin{array}{l}\text { b. Model II: adjusted for age }(<60, \geqq 60) \text {, race (Mexican American, other Hispanic, non-Hispanic white, non-Hispanic black, } \\
\text { other races), and BMI }(\leqq 25,25 \sim 30,>30) \text {. }\end{array}$} \\
\hline \multicolumn{7}{|c|}{$\begin{array}{l}\text { c. Model III: adjusted for age }(<60, \geqq 60) \text {, race (Mexican American, other Hispanic, non-Hispanic white, non-Hispanic black, } \\
\text { other races), BMI ( } \leqq 25,25 \sim 30,>30) \text {, ratio of family income to poverty }(<1.3,1.3 \sim 3.5,>3.5) \text {, education level (less than } \\
\text { high school, high school or general educational development), marital status (married or living with partner, living alone), } \\
\text { Charlson comorbidity index }(0,1,2345) \text {, physical activity (less than moderate, moderate, vigorous), smoking status (never, } \\
\text { former, current), alcohol drinking (none, moderate, heavy). }\end{array}$} \\
\hline
\end{tabular}


Table 5

Association between urinary PAHs and MUI among US adults, NHANES 2007 2016

\begin{tabular}{|c|c|c|c|c|c|c|}
\hline \multirow[t]{2}{*}{ Exposure } & \multicolumn{2}{|l|}{ Model la } & \multicolumn{2}{|l|}{ Model IIb } & \multicolumn{2}{|l|}{ Model IIIC } \\
\hline & OR $(95 \% \mathrm{Cl})$ & $\begin{array}{l}p \\
\text { value }\end{array}$ & OR $(95 \% \mathrm{Cl})$ & $\begin{array}{l}\mathrm{p} \\
\text { value }\end{array}$ & OR $(95 \% \mathrm{Cl})$ & $\mathrm{p}_{\text {value }}$ \\
\hline \multicolumn{7}{|c|}{ 1-hydroxynapthalene } \\
\hline quantile 1 & 1.000 & - & 1.000 & - & 1.000 & - \\
\hline quantile 2 & $0.941(0.797,1.111)$ & 0.472 & $1.101(0.921,1.315)$ & 0.291 & $1.098(0.917,1.314)$ & 0.311 \\
\hline quantile 3 & $0.940(0.796,1.110)$ & 0.464 & $1.097(0.917,1.312)$ & 0.311 & $1.075(0.893,1.294)$ & 0.444 \\
\hline quantile 4 & $0.927(0.785,1.095)$ & 0.371 & $1.263(1.048,1.523)$ & 0.014 & $1.099(0.884,1.365)$ & 0.397 \\
\hline$p$ for trend & & 0.547 & & 0.021 & & 0.681 \\
\hline \multicolumn{7}{|c|}{ 2-hydroxynapthalene } \\
\hline quantile 1 & 1.000 & - & 1.000 & - & 1.000 & - \\
\hline quantile 2 & $0.858(0.728,1.012)$ & 0.069 & $1.115(0.934,1.331)$ & 0.228 & $1.063(0.888,1.274)$ & 0.505 \\
\hline quantile 3 & $0.898(0.763,1.057)$ & 0.196 & $1.359(1.131,1.632)$ & 0.001 & $1.235(1.019,1.498)$ & 0.032 \\
\hline quantile 4 & $0.806(0.683,0.952)$ & 0.011 & $1.470(1.204,1.795)$ & $\begin{array}{l}< \\
0.001\end{array}$ & $1.230(0.976,1.551)$ & 0.080 \\
\hline$p$ for trend & & 0.040 & & $\begin{array}{l}< \\
0.001\end{array}$ & & 0.134 \\
\hline
\end{tabular}

3-hydroxyfluorene

\begin{tabular}{|c|c|c|c|c|c|c|}
\hline quantile 1 & 1.000 & - & 1.000 & - & 1.000 & - \\
\hline quantile 2 & $0.802(0.685,0.939)$ & 0.006 & $1.028(0.863,1.225)$ & 0.754 & $1.014(0.849,1.211)$ & 0.879 \\
\hline quantile 3 & $0.599(0.505,0.709)$ & $\begin{array}{l}< \\
0.001\end{array}$ & $0.920(0.759,1.115)$ & 0.395 & $0.875(0.718,1.068)$ & 0.190 \\
\hline quantile 4 & $0.710(0.604,0.835)$ & $\begin{array}{l}< \\
0.001\end{array}$ & $1.227(1.013,1.487)$ & 0.037 & $1.045(0.810,1.348)$ & 0.735 \\
\hline$p$ for trend & & 0.015 & & 0.006 & & 0.452 \\
\hline
\end{tabular}

2-hydroxyfluorene

\begin{tabular}{|lllllll|} 
quantile 1 & 1.000 & - & 1.000 & - & 1.000 & - \\
\hline quantile 2 & $0.833(0.710,0.977)$ & 0.025 & $1.101(0.921,1.316)$ & 0.292 & $1.057(0.881,1.268)$ & 0.551 \\
quantile 3 & $0.686(0.581,0.810)$ & $<$ & $1.059(0.873,1.285)$ & 0.560 & $0.993(0.811,1.215)$ & 0.942 \\
& & 0.001 & & & &
\end{tabular}

abbr. PAH, polycyclic aromatic hydrocarbons; $\mathrm{MUI}$, mixed urinary incontinence; OR, odds ratio; $\mathrm{Cl}$, confidence interval

a. Model I: unadjusted model

b. Model II: adjusted for age $(<60, \geqq 60)$, race (Mexican American, other Hispanic, non-Hispanic white, non-Hispanic black, and other races), and $\mathrm{BMI}(\leqq 25,25 \sim 30,>30)$.

c. Model III: adjusted for age $(<60, \geqq 60)$, race (Mexican American, other Hispanic, non-Hispanic white, non-Hispanic black, other race), $\mathrm{BMI}(\leqq 25,25 \sim 30,>30)$, ratio of family income to poverty $(<1.3,1.3 \sim 3.5,>3.5)$, education level (less than high school, high school or general educational development), marital status (married or living with partner, living alone), Charlson comorbidity index $(0,1,2345)$, physical activity (less than moderate, moderate, vigorous), smoking status (never, former, current), alcohol drinking (none, moderate, heavy). 


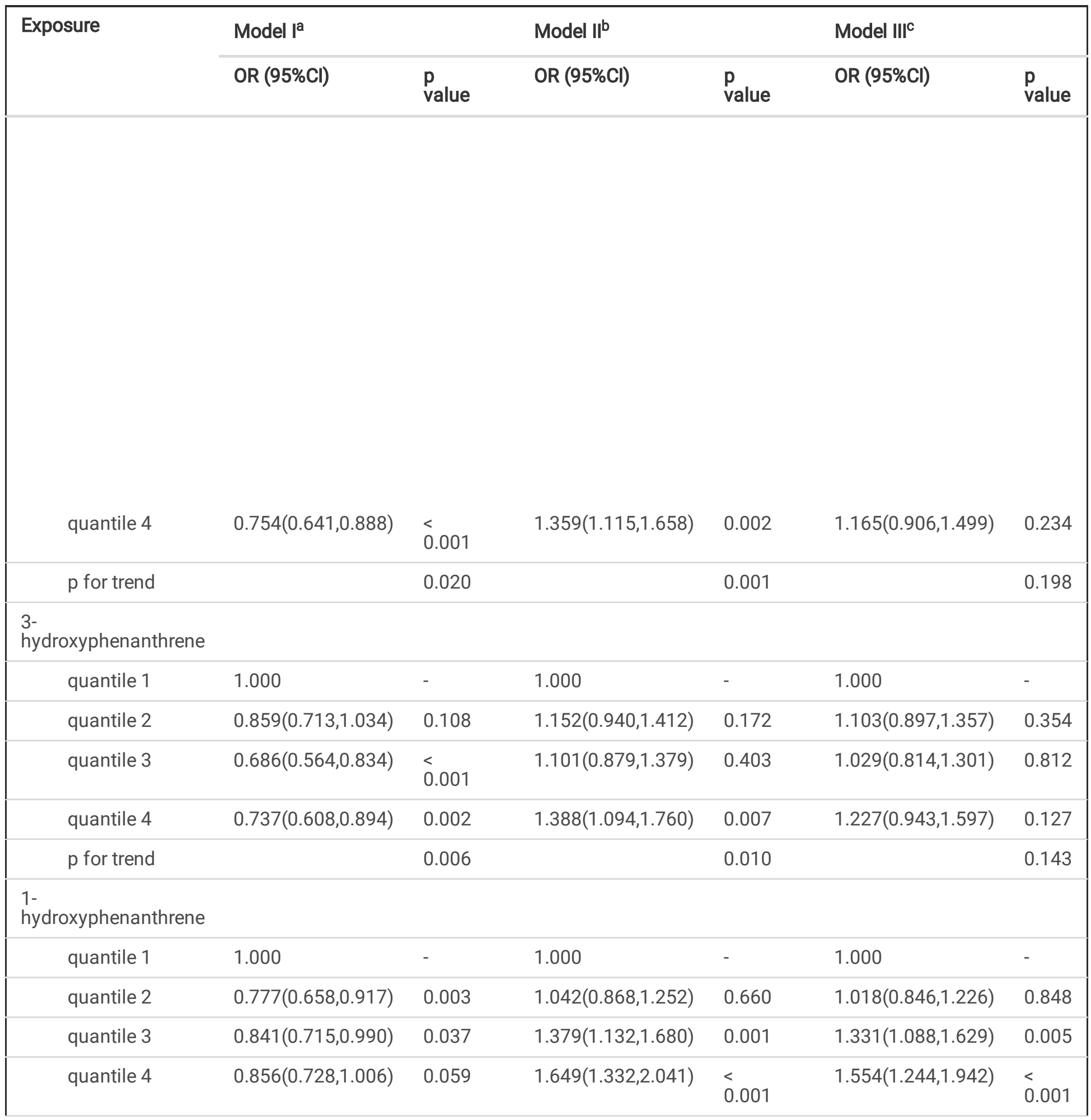

abbr. PAH, polycyclic aromatic hydrocarbons; $\mathrm{MUI}$, mixed urinary incontinence; OR, odds ratio; $\mathrm{Cl}$, confidence interval

a. Model I: unadjusted model

b. Model II: adjusted for age $(<60, \geqq 60)$, race (Mexican American, other Hispanic, non-Hispanic white, non-Hispanic black, and other races), and $\mathrm{BMI}(\leqq 25,25 \sim 30,>30)$.

c. Model III: adjusted for age $(<60, \geqq 60)$, race (Mexican American, other Hispanic, non-Hispanic white, non-Hispanic black, other race), BMI ( $\leqq 25,25 \sim 30,>30)$, ratio of family income to poverty $(<1.3,1.3 \sim 3.5,>3.5)$, education level (less than high school, high school or general educational development), marital status (married or living with partner, living alone), Charlson comorbidity index $(0,1,2345)$, physical activity (less than moderate, moderate, vigorous), smoking status (never, former, current), alcohol drinking (none, moderate, heavy). 


\begin{tabular}{|c|c|c|c|c|c|c|}
\hline \multirow[t]{2}{*}{ Exposure } & \multicolumn{2}{|l|}{ Model la } & \multicolumn{2}{|l|}{ Model II } & \multicolumn{2}{|l|}{ Model IIII } \\
\hline & OR $(95 \% \mathrm{Cl})$ & $\begin{array}{l}\mathrm{p} \\
\text { value }\end{array}$ & OR (95\%Cl) & $\begin{array}{l}\mathbf{p} \\
\text { value }\end{array}$ & OR $(95 \% \mathrm{Cl})$ & $\begin{array}{l}\mathrm{p} \\
\text { value }\end{array}$ \\
\hline$p$ for trend & & 0.350 & & $\begin{array}{l}< \\
0.001\end{array}$ & & $\begin{array}{l}< \\
0.001\end{array}$ \\
\hline \multicolumn{7}{|c|}{$\begin{array}{l}\text { 2- } \\
\text { hydroxyphenanthrene }\end{array}$} \\
\hline quantile 1 & 1.000 & - & 1.000 & - & 1.000 & - \\
\hline quantile 2 & $0.927(0.768,1.120)$ & 0.433 & $1.295(1.052,1.595)$ & 0.015 & $1.246(1.008,1.541)$ & 0.042 \\
\hline quantile 3 & $0.823(0.678,0.998)$ & 0.048 & $1.391(1.104,1.752)$ & 0.005 & $1.308(1.030,1.661)$ & 0.028 \\
\hline quantile 4 & $0.798(0.657,0.969)$ & 0.023 & $1.557(1.215,1.995)$ & $<0.001$ & $1.387(1.062,1.812)$ & 0.017 \\
\hline $\mathrm{p}$ for trend & & 0.024 & & 0.004 & & 0.081 \\
\hline \multicolumn{7}{|l|}{ 1-hydroxypyrene } \\
\hline quantile 1 & 1.000 & - & 1.000 & - & 1.000 & - \\
\hline quantile 2 & $0.920(0.784,1.080)$ & 0.308 & $1.124(0.950,1.329)$ & 0.172 & $1.103(0.930,1.308)$ & 0.262 \\
\hline quantile 3 & $0.802(0.678,0.949)$ & 0.010 & $1.280(1.063,1.542)$ & 0.009 & $1.210(0.998,1.467)$ & 0.052 \\
\hline quantile 4 & $0.734(0.618,0.871)$ & $<.001$ & $1.476(1.207,1.805)$ & $<.001$ & $1.307(1.047,1.630)$ & 0.018 \\
\hline $\mathrm{p}$ for trend & & $<.001$ & & $<.001$ & & 0.035 \\
\hline \multicolumn{7}{|c|}{ 9-hydroxyfluorene } \\
\hline quantile 1 & 1.000 & - & 1.000 & - & 1.000 & - \\
\hline quantile 2 & $0.781(0.636,0.958)$ & 0.018 & $0.993(0.795,1.239)$ & 0.947 & $0.952(0.760,1.194)$ & 0.671 \\
\hline quantile 3 & $0.768(0.625,0.943)$ & 0.012 & $1.146(0.903,1.452)$ & 0.262 & $1.059(0.826,1.358)$ & 0.649 \\
\hline quantile 4 & $0.690(0.559,0.852)$ & $<.001$ & $1.167(0.902,1.509)$ & 0.240 & $0.981(0.733,1.313)$ & 0.898 \\
\hline$p$ for trend & & 0.004 & & 0.189 & & 0.949 \\
\hline \multicolumn{7}{|c|}{$\begin{array}{l}\text { 4- } \\
\text { hydroxyphenanthrene }\end{array}$} \\
\hline quantile 1 & 1.000 & - & 1.000 & - & 1.000 & - \\
\hline quantile 2 & $0.866(0.653,1.149)$ & 0.319 & $1.110(0.816,1.509)$ & 0.508 & $1.138(0.832,1.558)$ & 0.419 \\
\hline
\end{tabular}

abbr. PAH, polycyclic aromatic hydrocarbons; $\mathrm{MUI}$, mixed urinary incontinence; OR, odds ratio; $\mathrm{Cl}$, confidence interval

a. Model I: unadjusted model

b. Model II: adjusted for age $(<60, \geqq 60)$, race (Mexican American, other Hispanic, non-Hispanic white, non-Hispanic black, and other races), and $\mathrm{BMI}(\leqq 25,25 \sim 30,>30)$.

c. Model III: adjusted for age $(<60, \geqq 60)$, race (Mexican American, other Hispanic, non-Hispanic white, non-Hispanic black, other race), $\mathrm{BMI}(\leqq 25,25 \sim 30,>30)$, ratio of family income to poverty $(<1.3,1.3 \sim 3.5,>3.5)$, education level (less than high school, high school or general educational development), marital status (married or living with partner, living alone), Charlson comorbidity index $(0,1,2345)$, physical activity (less than moderate, moderate, vigorous), smoking status (never, former, current), alcohol drinking (none, moderate, heavy). 


\begin{tabular}{|c|c|c|c|c|c|c|}
\hline \multirow[t]{2}{*}{ Exposure } & \multicolumn{2}{|l|}{ Model la } & \multicolumn{2}{|l|}{ Model II } & \multicolumn{2}{|l|}{ Model IIIC } \\
\hline & OR $(95 \% \mathrm{Cl})$ & palue & OR $(95 \% \mathrm{Cl})$ & $\begin{array}{l}\text { p } \\
\text { value }\end{array}$ & OR $(95 \% \mathrm{Cl})$ & $\begin{array}{l}\text { p value } \\
\text { val }\end{array}$ \\
\hline quantile 3 & $0.886(0.668,1.176)$ & 0.403 & $1.313(0.948,1.819)$ & 0.102 & $1.400(0.996,1.968)$ & 0.053 \\
\hline quantile 4 & $0.749(0.558,1.004)$ & 0.053 & $1.326(0.924,1.902)$ & 0.126 & $1.392(0.937,2.066)$ & 0.101 \\
\hline$p$ for trend & & 0.077 & & 0.167 & & 0.167 \\
\hline \multicolumn{7}{|c|}{ abbr. PAH, polycyclic aromatic hydrocarbons; $\mathrm{MUI}$, mixed urinary incontinence; OR, odds ratio; $\mathrm{Cl}$, confidence interval } \\
\hline \multicolumn{7}{|c|}{ a. Model I: unadjusted model } \\
\hline \multicolumn{7}{|c|}{$\begin{array}{l}\text { b. Model II: adjusted for age }(<60, \geqq 60) \text {, race (Mexican American, other Hispanic, non-Hispanic white, non-Hispanic black, } \\
\text { and other races), and } \mathrm{BMI}(\leqq 25,25 \sim 30,>30) \text {. }\end{array}$} \\
\hline \multicolumn{7}{|c|}{$\begin{array}{l}\text { c. Model III: adjusted for age }(<60, \geqq 60) \text {, race (Mexican American, other Hispanic, non-Hispanic white, non-Hispanic black, } \\
\text { other race), BMI ( } \leqq 25,25 \sim 30,>30) \text {, ratio of family income to poverty }(<1.3,1.3 \sim 3.5,>3.5) \text {, education level (less than high } \\
\text { school, high school or general educational development), marital status (married or living with partner, living alone), } \\
\text { Charlson comorbidity index }(0,1,2345) \text {, physical activity (less than moderate, moderate, vigorous), smoking status (never, } \\
\text { former, current), alcohol drinking (none, moderate, heavy). }\end{array}$} \\
\hline
\end{tabular}

\section{Discussion}

In present study, we provide a new evidence for the association between PAHs and UI using the NHANES database from 2007 to 2016. This study suggested that increasing urine PAHs were possibly associated with the increasing prevalence of all three subtypes of Ul.

The biological mechanism of the association between PAHs and $\mathrm{UI}$ is still unclear. Thus, we can only propose a hypothesis for the underlying mechanism based on the available research evidence. Studies have shown that exposure to PAHs has certain effects on reproductive function and serum sex hormones levels (18-21). Associations between sex hormones and all three subtypes of UI were reported in other studies $(15 ; 22 ; 23)$. Thus, PAHs may potentially affect UI prevalence by changing levels of several sex hormones.

The significant association between almost all urine PAHs and SUI were described in the present study. The previous study of Augoulea et. al revealed the association between the reduction of serum estradiol (E2) and $\triangle 4$-Androstendione ( $\triangle 4 A$ ) levels and SUI (24). The decreasing level E2 was also observed in participants with increasing exposure of PAHs by Yang et. al (25). Ahn et al. reported that E2 and follicle-stimulating hormone (FSH) were related to urodynamics in female patients with SUI (23), which called for studies between PAHs exposure and urodynamic parameters in SUI patients.

This study has the following strengths. First, this study had a large sample size, including a total of 12,393 participants from 2007 to 2016 . Second, the sample selection in this study was relatively random, and the selected sample population was national representative. Thirdly, the data collection standards of this study were relatively uniform, and all the data related to this study were collected in accordance with the NHANES survey collection standards.

However, several limitations of this study also need to be acknowledged. First, this study was a cross-sectional study, which caused that it cannot be determined whether the causal relationship between PAHs and UI existed. Second, UI diagnosis was based on self-reported questionnaires, which may generate measurement bias. Thirdly, a single sample can only show the PAHs levels at a specific time, and the short half-lives of PAHs make it unable to account for persistent exposure and individual differences. Further cohort studies and experimental studies need to be conducted to verify the result of the present study.

\section{Conclusion}


Our study found a positive association between U-PAHs and all three subtypes of Ul risk. Almost all PAHs have positive relations with SUI. The results of this study need to be verified by further cohort studies and experimental studies.

\section{Abbreviations}

PAHs, polycyclic aromatic hydrocarbons; UI, urinary incontinence; SUI, stress urinary incontinence; UUl, urgency urinary incontinence; GC-HRMS, capillary gas chromatography combined with high resolution mass spectrometry; GC-MS/MS, isotope dilution gas chromatography/tandem mass spectrometry; online SPE-HPLC-MS/MS, isotope dilution online solid phase extraction high performance liquid chromatography/tandem mass spectrometry; MUI, mixed urinary incontinence; OR, odds ratio; $95 \% \mathrm{Cl}$, 95\% confidence intervals; NHANES, National Health and Nutrition Examination Survey; NCHS, National Center for Health Statistics; MECs, mobile examination centers; BMI, body mass index; SD, standard deviation; GM, geometric mean; LLOD, lower limit of detection; $\mathrm{E} 2$, estradiol; $\triangle 4 \mathrm{~A}, \triangle 4$-Androstendione; $\mathrm{FSH}$, follicle-stimulating hormone.

\section{Declarations}

Ethics Approval and Consent to Participate

The study was approved by Ethics Review Board of NCHS, and all participants had been required to sign an informed consent form before the study began.

Consent for Publication

Not applicable.

Availability of Data and Materials

The datasets generated and/or analysed during the current study are available in the National Health and Nutrition Examination Survey repository, [http://www.cdc.gov/nchs/nhanes.htm].

Competing Interests

The authors declare that they have no known competing financial interests or personal relationships that could have appeared to influence the work reported in this paper.

Fundings

The work was supported by the National key research and development program of China (Grant No.2017YFC0908003), National Natural Science Foundation of China (Grant No.81902578, 81974098), China Post-docotral Science Foundation (2017M612971), Post-doctoral Science Research Foundation of Sichuan University (2020SCU12041), Post-Doctor Research Project, West China Hospital, Sichuan University (2018HYBH085), National Clinical Research Center for Geriatrics, West China Hospital, Sichuan University (Z2018C01) and Xing-Lin Chen (EmpowerStats X\&Y Solutions, Inc, Boston, MA) for providing statistical methodology consultation.

Acknowledgement

Not applicable.

Author's Contributions

Zilong Zhang: Data collection; Methodology; Writing - original draft; Kun Jin: Methodology; Formal analysis; Writing - original draft; Shi Qiu: Methodology; Data curation; Writing - original draft; Xinyi Huang: Data collection, Table and figure preparation; Dan Hu: Data collection; Zhongyuan Jiang: Validation and Editing; Mingda Wang: Validation and Editing; Lu Yang: Methodology; Formal analysis; Tianhai Lin: Data collection; Supervision; Xianghong Zhou: Data collection; Di Jin: Data 
collection; Yu Zhan: Conceptualization, Methodology, and Reviewing; Qiang Wei: Conceptualization, Methodology, and Reviewing

\section{References}

1. Chen YY, Kao TW, Wang CC, Chen YJ, Wu CJ, et al. Polycyclic aromatic hydrocarbon metabolites and mortality risk in an adult population. Environ Geochem Health. 2021;43:37-45.

2. Chen YY, Kao TW, Wang CC, Chen YJ, Wu CJ, et al. Exposure to polycyclic aromatic hydrocarbons and risk of disability among an elderly population. Environ Sci Pollut Res Int. 2019;26:10719-26.

3. Alegbeleye 00, Opeolu BO, Jackson VA. Polycyclic Aromatic Hydrocarbons: A Critical Review of Environmental Occurrence and Bioremediation. Environ Manage. 2017;60:758-83.

4. Shiue I. Urinary polyaromatic hydrocarbons are associated with adult celiac disease and kidney stones: USA NHANES, 2011-2012. Environ Sci Pollut Res Int. 2016;23:3971-7.

5. DellaValle CT, Deziel NC, Jones RR, Colt JS, De Roos AJ, et al. Polycyclic aromatic hydrocarbons: determinants of residential carpet dust levels and risk of non-Hodgkin lymphoma. Cancer Causes Control. 2016;27:1-13.

6. Tfouni SAV, Reis RM, Kamikata K, Gomes FML, Morgano MA, Furlani RPZ. Polycyclic aromatic hydrocarbons in teas using QuEChERS and HPLC-FLD. Food Addit Contam Part B Surveill. 2018;11:146-52.

7. Zhang W, Yang J, Lv Y, Li S, Qiang M. Paternal benzo[a]pyrene exposure alters the sperm DNA methylation levels of imprinting genes in F0 generation mice and their unexposed F1-2 male offspring. Chemosphere. 2019;228:586-94.

8. Liu J, Zhang L, Winterroth LC, Garcia M, Weiman S, et al. 2013. Epigenetically mediated pathogenic effects of phenanthrene on regulatory T cells. J Toxicol 2013:967029.

9. Lukacz ES, Santiago-Lastra Y, Albo ME, Brubaker L. Urinary Incontinence in Women: A Review. Jama. 2017;318:1592-604.

10. Stothers $L$, Thom D, Calhoun E. Urologic diseases in America project: urinary incontinence in males-demographics and economic burden. J Urol. 2005;173:1302-8.

11. Howard F, Steggall M. Urinary incontinence in women: quality of life and help-seeking. Br J Nurs. 2010;19:742(4):6, 8-9.

12. Landefeld CS, Bowers BJ, Feld AD, Hartmann KE, Hoffman E, et al. 2008. National Institutes of Health state-of-the-science conference statement: prevention of fecal and urinary incontinence in adults. Ann Intern Med 148:449 - 58.

13. Thüroff JW, Abrams P, Andersson KE, Artibani W, Chapple CR, et al. EAU guidelines on urinary incontinence. Eur Urol. 2011;59:387-400.

14. Hu TW, Wagner TH, Bentkover JD, Leblanc K, Zhou SZ, Hunt T. Costs of urinary incontinence and overactive bladder in the United States: a comparative study. Urology. 2004;63:461-5.

15. Kim MM, Kreydin El. The Association of Serum Testosterone Levels and Urinary Incontinence in Women. J Urol. 2018;199:522-7.

16. Cardozo LD, Kelleher CJ. Sex hormones, the menopause and urinary problems. Gynecol Endocrinol. 1995;9:75-84.

17. Tao C, Fan Y, Niu R, Li Z, Qian H, et al. Urinary polycyclic aromatic hydrocarbons and sex hormones in children and adolescents: Evidence from NHANES. Ecotoxicol Environ Saf. 2021;216:112215.

18. Luderer U, Christensen F, Johnson WO, She J, Ip HS, et al. Associations between urinary biomarkers of polycyclic aromatic hydrocarbon exposure and reproductive function during menstrual cycles in women. Environ Int. 2017;100:110-20.

19. Wang L, Hu W, Xia Y, Wang X. Associations between urinary polycyclic aromatic hydrocarbon metabolites and serum testosterone in U.S. adult males: National Health and nutrition examination survey 2011-2012. Environ Sci Pollut Res Int. 2017;24:7607-16.

20. Yin S, Tang M, Chen F, Li T, Liu W. Environmental exposure to polycyclic aromatic hydrocarbons (PAHs): The correlation with and impact on reproductive hormones in umbilical cord serum. Environ Pollut. 2017;220:1429-37.

21. Han Y, Xia Y, Zhu P, Qiao S, Zhao R, et al. Reproductive hormones in relation to polycyclic aromatic hydrocarbon (PAH) metabolites among non-occupational exposure of males. Sci Total Environ. 2010;408:768-73. 
22. Grady D, Brown JS, Vittinghoff E, Applegate W, Varner E, Snyder T. Postmenopausal hormones and incontinence: the Heart and Estrogen/Progestin Replacement Study. Obstet Gynecol. 2001;97:116-20.

23. Ahn KH, Kim T, Hur JY, Kim SH, Lee KW, Kim YT. Relationship between serum estradiol and follicle-stimulating hormone levels and urodynamic results in women with stress urinary incontinence. Int Urogynecol J. 2011;22:731-7.

24. Augoulea A, Sioutis D, Rizos D, Panoulis C, Triantafyllou N, et al. Stress urinary incontinence and endogenous sex steroids in postmenopausal women. Neurourol Urodyn. 2017;36:121-5.

25. Yang P, Sun H, Gong YJ, Wang YX, Liu C, et al. Repeated measures of urinary polycyclic aromatic hydrocarbon metabolites in relation to altered reproductive hormones: A cross-sectional study in China. Int J Hyg Environ Health. 2017;220:1340-6.

\section{Figures}

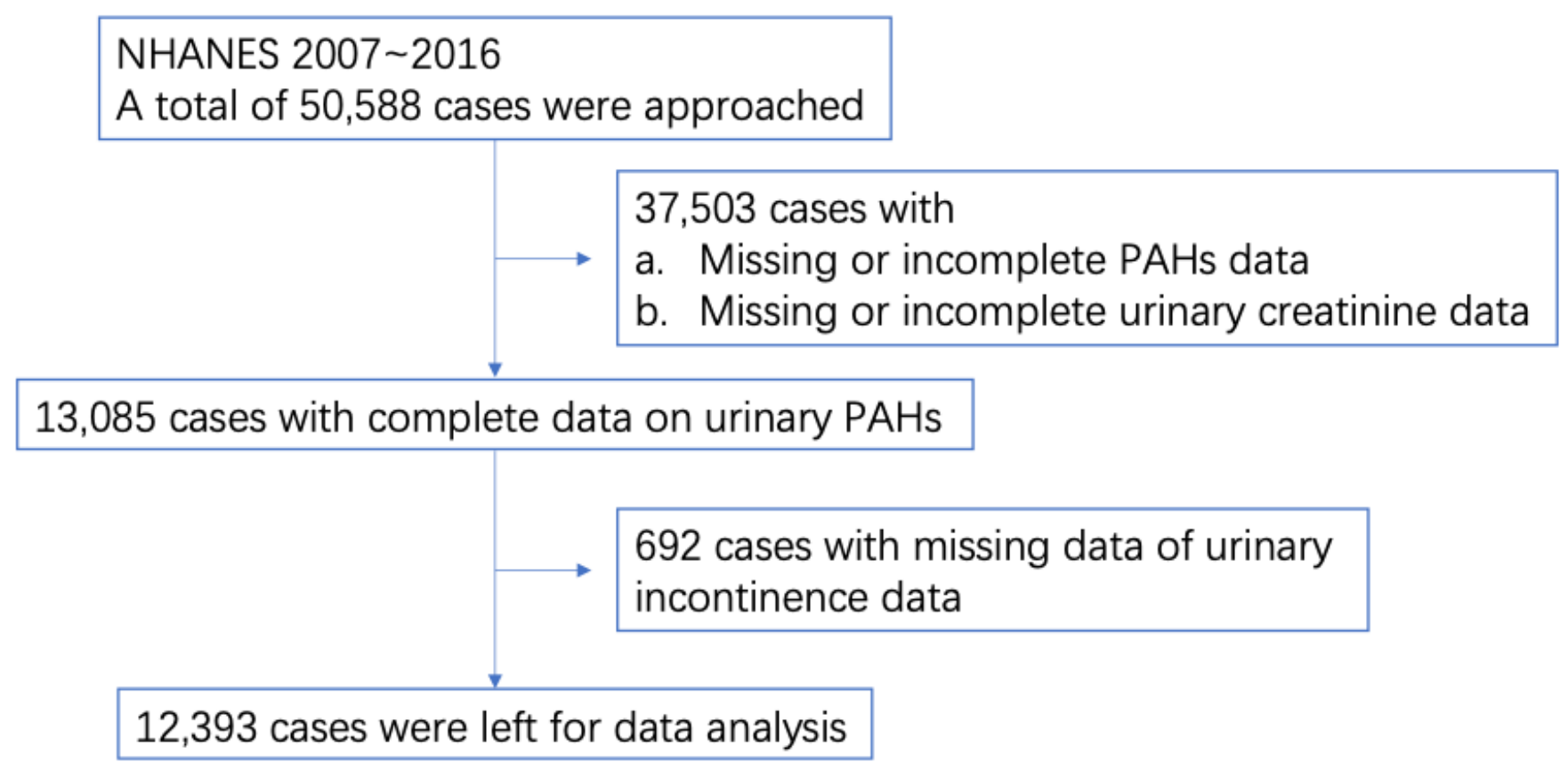

\section{Figure 1}

In the NHANES database from 2007 to 2016, a total of 50,588 participants aged over 20 were enrolled in the survey, among which 13,085 participants with detectable level of urinary PHAs metabolites were included. 692 participants were excluded due to lack of UI information. Finally, a total of 12,393 adults over 20 years of age were analyzed. Figure 1 showed the flowchart of participants selection.

\section{Supplementary Files}

This is a list of supplementary files associated with this preprint. Click to download.

- GraphicAbstract.pdf 